\title{
Discovery of a planetary nebula surrounding the symbiotic star DT Serpentis
}

\author{
U. Munari ${ }^{1}$, R. L. M. Corradi ${ }^{2,3}$, A. Siviero ${ }^{4}$, L. Baldinelli ${ }^{5}$, and A. Maitan ${ }^{5}$ \\ 1 INAF Osservatorio Astronomico di Padova, 36012 Asiago (VI), Italy \\ e-mail: ulisse.munari@oapd.inaf.it \\ 2 Instituto de Astrofisica de Canarias, 38200 La Laguna, Tenerife, Spain \\ 3 Departamento de Astrofísica, Universidad de La Laguna, 38206 La Laguna, Tenerife, Spain \\ ${ }^{4}$ Department of Physics and Astronomy, University of Padova, 36012 Asiago (VI), Italy \\ 5 ANS Collaboration, c/o Astronomical Observatory, 36012 Asiago (VI), Italy
}

Received 13 May 2013 / Accepted 11 August 2013

\begin{abstract}
We report the discovery of a planetary nebula centered on the poorly studied symbiotic binary star DT Ser. In a few other symbiotic stars spatially resolved nebulae have been discovered as well, but only one of them probably is a genuine planetary nebula, while the others are likely to originate in complex mass-ejection episodes from the interacting binary central stars that are possibly related to nova-like outbursts. The rim of the planetary nebula around DT Ser is severely distorted toward a brighter star 5 arcsec away. In infrared WISE data, this star shows the presence of a detached cold-dust shell similar to those observed in post-AGB stars. The apparent association of the symbiotic star and its planetary nebula with the nearby possible post-AGB object is discussed. We also discuss the sparse and conflicting literature data that could support an observed variability of the surface brightness of the planetary nebula. The puzzling and intriguing characteristics displayed by DT Ser are surely worth additional and more detailed investigations.
\end{abstract}

Key words. binaries: symbiotic - planetary nebulae: general - stars: individual: DT Ser - stars: AGB and post-AGB

\section{Introduction}

Symbiotic stars are binaries composed of a late-type giant/supergiant and a white dwarf (WD) companion. The WD is bright $\left(10^{3} L_{\odot}\right)$ and hot $\left(10^{5} \mathrm{~K}\right)$ and typically experiences surface stable H-burning of material accreted from the cool giant. The hard-radiation field ionizes part of the wind of the giant, giving rise to a rich and high ionization emission line spectrum and a bright Balmer continuum in emission, which are superimposed on the absorption spectrum of the cool giant. Given the usually large distances to symbiotic stars and the limited extent of the region of the cool giant wind ionized by the WD radiation field, nearly all known symbiotic stars appear as point-like when viewed with a telescope.

Spatially resolved nebulae have so far been discovered only around a few of the about 220 symbiotic stars listed in the cata$\log$ by Belczyński et al. (2000). The 3D morpho-kinematic structure of some of them suggests a ballistic launch by some sort of explosive event (Corradi 2003). The largest and more spectacular of these nebulae are seen in symbiotic binaries where the cool giant is a Mira variable: R Aqr (Lampland 1922; Solf \& Ulrich 1985; Paresce \& Hack 1994), Hen 2-104 (Schwarz et al. 1989; Corradi et al. 2001a; Santander-García et al. 2008), BI Cru (Schwarz \& Corradi 1992), Hen 2-147 (Munari \& Patat 1993; Santander-García et al. 2007), V1016 Cyg (Solf 1983; Corradi et al. 1999) and HM Sge (Solf 1984; Corradi et al. 1999). Nebulae have also been discovered around symbiotic stars that harbor non-Mira cool giants: AS 201 (Schwarz 1991) and CH Cyg (Corradi et al. 2001b). Bipolar planetary nebulae like M 2-9 (Corradi et al. 2011) and Mz 3 (Guerrero et al. 2004; Santander-García et al. 2004) that may possibly host a symbiotic nucleus (Schmeja \& Kimeswenger 2001) are relevant bridges between symbiotic binaries and aspherical planetary nebulae.

The discovery of extended nebulae around symbiotic stars, for its rarity, is an interesting occurrence worth more detailed investigation. The presence and nature of such nebulae are relevant in addressing the age and formation paths for symbiotic stars, their relation to planetary nebulae with binary nuclei, and the feasibility of the symbiotic star channel for the progenitors of type Ia supernovae. In this paper we report our discovery of a low surface-brightness planetary nebula, 11.4 arcsec in diameter, centered on the symbiotic star DT Ser.

\section{DT Serpentis}

DT Ser is a poorly studied variable, reported by the General Catalog of Variable Stars (GCVS) to vary irregularly between magnitude 13.2 and 13.9 in the blue photographic system, following early discovery reports of 1957 . Jurdana-Šepić \& Munari (2010) found DT Ser stable around mean values of $B=13.17$ and $I_{\mathrm{C}}=11.63$ on Asiago archive plates exposed between 1972 and 1977. USNO-B first- and second-epoch magnitudes of DT Ser are $B=14.11$ and $B=13.56$, respectively.

Bond (1978, hereafter B78) reported in his spectroscopic survey of high galactic latitude blue variables that DT Ser displayed a G-type absorption spectrum and superimposed emission lines, in particular from [O III]. He suggested a classification as a probable symbiotic binary, noted a stellar appearance, and observed the presence to the southwest of what he described as a slightly fainter companion 5 arcsec away (which is merged with the primary into an unresolved and elongated single stellar image on Palomar Sky Survey plates). In the following we 

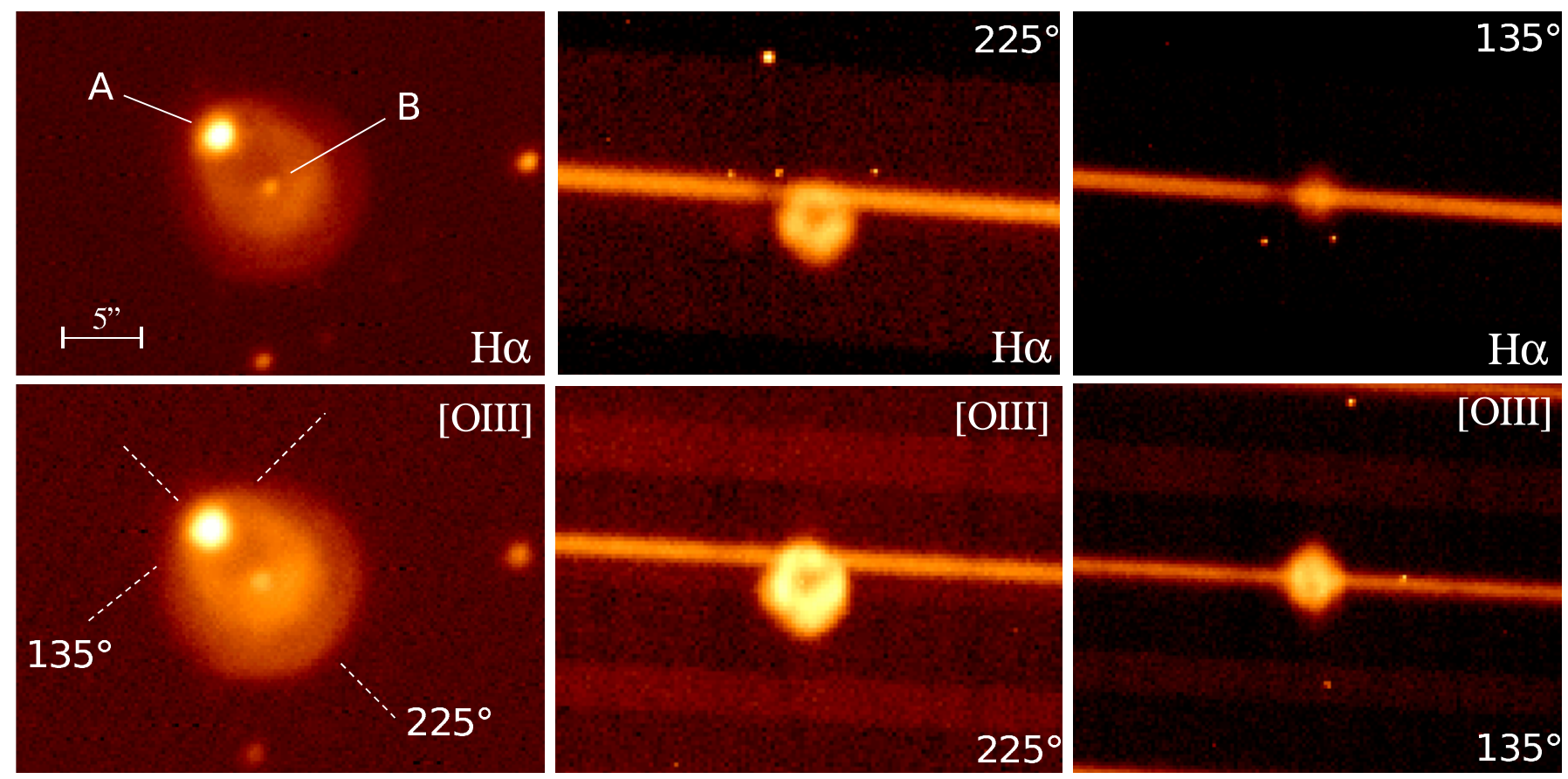

Fig. 1. Left: NOT images (in $\mathrm{H} \alpha$ and [O III] $5007 \AA$ light) of the planetary nebula we discovered around the symbiotic star DT Ser. North is up, east to the left, and the overall diameter of the nebula is 11.4 arcsec. Stars A and B (5 arcsec away) that are mentioned in the text are identified. The $225^{\circ}$ and $135^{\circ}$ slit positions, centered on star A, are also identified. Positions S-E and N-W are referred to in Fig. 4 and the text (Sect. 5). Center: portion of the original 2D Echelle spectra for the $225^{\circ}$ slit position centered on $\mathrm{H} \alpha$ and [O III] $5007 \AA$. Right: the same for the $135^{\circ}$ slit position.

call "A" the star identified by B78 as the symbiotic star, and "B" the fainter companion 5 arcsec away (identified in Fig. 1).

Cieslinski et al. (1997, hereafter C97) obtained $U B V R_{\mathrm{C}} I_{\mathrm{C}}$ photometry and low-resolution spectroscopy of DT Ser. In contrast to B78, C97 found no emission lines associated to star A and instead determined that the emission lines (He II, He I, [O III]) were associated with star B, which they identified as the symbiotic star. C97 reported photoelectric photometry of star B as $V=15.40, U-B=-0.59, B-V=+0.51, V-R_{\mathrm{C}}=-0.06$, and $R_{\mathrm{C}}-I_{\mathrm{C}}=+0.10, \Delta V=2.6$ mag fainter than star A which C97 measured at $V=12.8$ and $B-V=+0.76$. PSF-fitting photometry on CCD images by Henden \& Munari (2001, 2008, hereafter HM01 and HM08 respectively) measured star B at $V=16.213$, $U-B=-0.820, B-V=+0.371, V-R_{\mathrm{C}}=-0.055$, and $R_{\mathrm{C}}-I_{\mathrm{C}}=+0.11$, and star $\mathrm{A}$ at $V=12.772, U-B=+0.109$, $B-V=+0.778, V-R_{\mathrm{C}}=+0.462$, and $R_{\mathrm{C}}-I_{\mathrm{C}}=+0.475$. The difference in magnitude between stars $\mathrm{A}$ and $\mathrm{B}$ as measured by HM08 is $\Delta V=3.4$, about 0.8 mag higher than derived by $\mathrm{C} 97$.

Neither B78 or C97 noticed any spatially resolved emission line on their spectra, in spite of the long-slit instrumental setup and the good seeing conditions they must have enjoyed to be able to observe star B separately from star A. In sharp contrast, we first noticed the planetary nebula around DT Ser directly at the telescope while cursorily inspecting the raw 2D files of the spectra discussed in this paper: emission lines of uniform spatial intensity were extending to one side of the stellar spectrum for several times the seeing. At the time of our observations, no trace of the B star was noted on the TV guiding system of the telescopes, indicating that it was several magnitudes fainter than star A.

\section{Imaging}

After the spectroscopic discovery of the spatially extended emission lines, narrow-band images of DT Ser were obtained at the $2.6 \mathrm{~m}$ Nordic Optical Telescope (NOT) with the ALFOSC instrument on 30 March 2013. The filter central wavelengths and full width at half maximum (FWHM), and the corresponding selected nebular emission lines, were 5007/30 ([O III] 5007) and 6563/30 $\AA$ (H $\alpha$ with some contamination of the [N II] 6548, 6583 doublet, which is very weak in in DT Ser). Exposure time was $5 \mathrm{~min}$ in each filter. The spatial scale of ALFOSC is $0 . ' 19$ pix $^{-1}$ and the seeing was 0.'7 FWHM for the $\mathrm{H} \alpha$ images and 1.' 0 for [O III].

The narrow-band images of DT Ser in Fig. 1 reveal the details of the extended nebula, which has a diameter of 11.4 arcsec. Its morphology is very similar in the $\mathrm{H} \alpha$ and [O III] filters and consists of an inner, brighter rim and an outer, fainter attached shell. This is a typical configuration of planetary nebulae, in which the formation of the inner rim is ascribed to the action of a fast post-AGB wind impinging on the slower, denser AGB wind. The wind interaction creates a hot inner bubble that compresses gas and drives the expansion of the rim. The attached shell, which generally has a linearly decreasing surface brightness profile (as in the case of DT Ser), is instead the result of the passage of the photo-ionization front produced by the central star through undisturbed AGB wind (see e.g. Schönberner et al. 2005).

But the peculiar aspect of the nebula around DT Ser is that the rim is severely distorted. Its SE side is brighter and closer to the central star, while the NE part is fainter and elongated toward star A, giving the impression that it might be related to it.

\section{Photometric monitoring}

Since 2007, we have regularly observed DT Ser with the ANS Collaboration telescope n. 020 as part of the monitoring of known symbiotic stars described by Munari et al. (2012a) and Munari \& Moretti (2012). The telescope is a 0.40-m f/5 Newton reflector equipped with a HiSis $23 \mathrm{ME} \mathrm{CCD}, 768 \times 1157$ array, 
U. Munari et al.: Discovery of a planetary nebula around DT Ser

Table 1. Our photometry of DT Ser (merged images of stars A and B) and the corresponding total error budget.

\begin{tabular}{|c|c|c|c|c|c|c|c|c|c|c|c|c|c|c|c|c|c|}
\hline \multirow{2}{*}{$\frac{\text { Date (UT) }}{20070714.442}$} & \multicolumn{2}{|c|}{ V } & \multicolumn{2}{|c|}{$B-V$} & \multicolumn{2}{|c|}{$V-R_{\mathrm{C}}$} & \multicolumn{2}{|c|}{$V-I_{\mathrm{C}}$} & \multirow{2}{*}{$\begin{array}{l}\text { Date (UT) } \\
20090811.362\end{array}$} & \multicolumn{2}{|c|}{ V } & \multicolumn{2}{|c|}{$B-V$} & \multicolumn{2}{|c|}{$V-R_{\mathrm{C}}$} & \multicolumn{2}{|c|}{$V-I_{\mathrm{C}}$} \\
\hline & 12.755 & \pm 0.011 & 0.823 & \pm 0.007 & 0.455 & \pm 0.006 & 0.928 & \pm 0.007 & & 12.740 & \pm 0.009 & 0.725 & \pm 0.011 & 0.472 & \pm 0.012 & 0.967 & \pm 0.014 \\
\hline 20070716.423 & 12.735 & 0.013 & 0.799 & 0.006 & 0.445 & 0.007 & 0.916 & 0.007 & 20090816.362 & 12.744 & 0.010 & 0.702 & 0.041 & 0.477 & 0.012 & 0.951 & 0.012 \\
\hline 20070720.366 & 12.761 & 0.011 & 0.782 & 0.015 & 0.474 & 0.008 & 0.948 & 0.008 & 20090823.349 & 12.773 & 0.006 & 0.745 & 0.012 & 0.491 & 0.008 & 0.987 & 0.013 \\
\hline 20070723.357 & 12.715 & 0.008 & 0.794 & 0.012 & 0.438 & 0.007 & 0.904 & 0.005 & 20090830.322 & 12.788 & 0.008 & 0.796 & 0.008 & 0.494 & 0.005 & 0.993 & 0.013 \\
\hline 20070726.387 & 12.797 & 0.009 & 0.806 & 0.015 & 0.481 & 0.006 & 0.962 & 0.005 & 20090908.305 & 12.744 & 0.011 & 0.752 & 0.025 & 0.481 & 0.015 & 0.962 & 0.012 \\
\hline 20070801.355 & 12.777 & 0.014 & 0.687 & 0.014 & 0.470 & 0.009 & 0.958 & 0.011 & 20090913.303 & 12.782 & 0.010 & 0.732 & 0.013 & 0.495 & 0.010 & 0.981 & 0.009 \\
\hline 20070804.355 & 12.791 & 0.010 & 0.762 & 0.010 & 0.455 & 0.005 & 0.943 & 0.005 & 20100531.433 & 12.755 & 0.016 & 0.773 & 0.028 & & & 0.970 & 0.016 \\
\hline 20070814.327 & 12.762 & 0.011 & 0.716 & 0.017 & 0.438 & 0.007 & 0.929 & 0.006 & 20100606.394 & 12.755 & 0.015 & 0.764 & 0.024 & & & 0.962 & 0.011 \\
\hline 20070825.306 & 12.793 & 0.012 & 0.804 & 0.011 & 0.465 & 0.010 & 0.965 & 0.011 & 20100618.426 & 12.757 & 0.010 & 0.769 & 0.013 & 0.480 & 0.012 & 0.957 & 0.009 \\
\hline 20070905.299 & 12.731 & 0.006 & 0.805 & 0.019 & 0.452 & 0.006 & 0.886 & 0.004 & 710.386 & 12.769 & 0.013 & 0.757 & 0.006 & 0.489 & 0.010 & 0.990 & 0.011 \\
\hline 20070913.307 & 12.756 & 0.008 & 0.776 & 0.007 & 0.459 & 0.005 & 0.975 & 0.006 & 20100716.423 & 12.760 & 0.011 & 0.775 & 0.010 & 0.494 & 0.009 & 1.009 & 0.022 \\
\hline 20070919.294 & 12.773 & 0.007 & 0.776 & 0.010 & 0.445 & 0.007 & 0.945 & 0.006 & 20100722.373 & 12.753 & 0.009 & 0.800 & 0.017 & 0.446 & 0.011 & 0.917 & 0.010 \\
\hline 20071001.265 & 12.737 & 0.009 & 0.781 & 0.009 & 0.450 & 0.007 & 0.938 & 0.005 & 20100730.429 & 12.770 & 0.009 & 0.775 & 0.005 & 0.478 & 0.005 & 0.986 & 0.008 \\
\hline 20071007.270 & 12.778 & 0.007 & 0.742 & 0.007 & 0.475 & 0.008 & 0.961 & 0.006 & 20100808.419 & 12.804 & 0.004 & & & 0.481 & 0.010 & 0.954 & 0.019 \\
\hline 20071019.232 & 12.805 & 0.012 & 0.799 & 0.007 & 0.478 & 0.008 & 0.954 & 0.008 & 20100822.368 & 12.740 & 0.006 & 0.783 & 0.013 & 0.488 & 0.021 & 0.924 & 0.012 \\
\hline 20080623.380 & & & 0.763 & & 0.460 & & & & 20100825.355 & 12.746 & 0.009 & 0.862 & 0.018 & 0.432 & 0.011 & 0.947 & 0.017 \\
\hline 20080628.406 & 12.760 & 0.009 & 0.764 & 0.011 & 0.449 & 0.006 & 0.924 & 0.014 & 20110621.391 & 12.727 & 0.012 & 0.784 & 0.014 & 0.444 & 0.008 & 0.942 & 0.007 \\
\hline 20080705.401 & 12.746 & 0.021 & 0.828 & 0.026 & 0.441 & 0.008 & 0.907 & 0.009 & 20110628.396 & 12.737 & 0.012 & 0.792 & 0.013 & 0.442 & 0.013 & 0.911 & 0.011 \\
\hline 20080719.445 & 12.755 & 0.006 & 0.808 & 0.009 & 0.454 & 0.006 & 0.903 & 0.012 & 20110718.374 & 12.692 & 0.013 & 0.752 & 0.023 & 0.460 & 0.015 & 0.924 & 0.009 \\
\hline 20080727.360 & 12.745 & 0.010 & 0.752 & 0.027 & 0.476 & 0.005 & 0.954 & 0.007 & 20110821.361 & 12.775 & 0.019 & 0.778 & 0.025 & 0.398 & 0.019 & 0.925 & 0.024 \\
\hline 20080802.351 & 12.777 & 0.008 & 0.709 & 0.013 & 0.501 & 0.006 & 0.966 & 0.010 & 20110828.355 & 12.761 & 0.021 & 0.767 & 0.024 & 0.450 & 0.025 & 0.934 & 0.023 \\
\hline 20080807.327 & 12.759 & 0.011 & 0.789 & 0.022 & 0.444 & 0.008 & 0.921 & 0.016 & 20110909.333 & 12.737 & 0.010 & 0.808 & 0.007 & 0.456 & 0.003 & 0.917 & 0.006 \\
\hline 20080820.348 & 12.750 & 0.016 & 0.747 & 0.026 & 0.459 & 0.013 & 0.947 & 0.017 & 20111002.290 & 12.728 & 0.014 & 0.781 & 0.008 & 0.462 & 0.005 & 0.917 & 0.010 \\
\hline 20080825.349 & 12.765 & 0.020 & 0.728 & 0.026 & 0.458 & 0.007 & 0.924 & 0.011 & 20120622.422 & 12.747 & 0.007 & 0.774 & 0.003 & 0.556 & 0.004 & 0.936 & 0.008 \\
\hline 20080830.320 & 12.747 & 0.008 & 0.772 & 0.013 & 0.444 & 0.007 & 0.931 & 0.019 & 20120713.410 & 12.723 & 0.007 & 0.782 & 0.008 & 0.436 & 0.005 & 0.926 & 0.012 \\
\hline 20090718.394 & 12.749 & 0.005 & 0.807 & 0.013 & 0.469 & 0.004 & 0.942 & 0.007 & 20120807.393 & 12.749 & 0.008 & 0.766 & 0.011 & 0.457 & 0.006 & 0.955 & 0.013 \\
\hline 20090724.380 & 12.767 & 0.008 & 0.756 & 0.036 & 0.482 & 0.006 & 0.946 & 0.006 & 20120820.364 & 12.733 & 0.009 & 0.787 & 0.009 & 0.462 & 0.004 & 0.924 & 0.005 \\
\hline 20090730.376 & 12.757 & 0.005 & 0.760 & 0.009 & 0.469 & 0.005 & 0.976 & 0.009 & 20120908.335 & 12.762 & 0.008 & 0.766 & 0.009 & 0.459 & 0.008 & 0.929 & 0.007 \\
\hline 20090804.375 & 12.753 & 0.006 & 0.778 & 0.010 & 0.472 & 0.010 & 0.977 & 0.018 & & & & & & & & & \\
\hline
\end{tabular}

operated in binned mode for a scale on the sky of $1.24^{\prime \prime} \mathrm{pix}^{-1}$, and $B V R_{\mathrm{C}} I_{\mathrm{C}}$ filters from Schuler. With this telescope and typical seeing conditions, stars $\mathrm{A}$ and $\mathrm{B}$ are merged into an unresolved single stellar image. Instrumental set-up and parameters of the photometric reduction (radius aperture, sky annulus well external to the nebula) have been kept strictly stable during the observing campaign, to avoid introducing spurious effects. Our aperture photometry was calibrated against the local photometric sequence of HM01. It is given in Table 1 and refers to the integrated light of stars A and B.

The photometric evolution is presented in Fig. 2, which indicates for all bands a steady brightening with time of the blended image of stars A + B over the 1883 day interval covered by the data in Table 1 . The brightening amounts to $\Delta B=-0.026$, $\Delta V=-0.021, \Delta R_{\mathrm{C}}=-0.024$ and $\Delta I_{\mathrm{C}}=-0.026 \mathrm{mag}$. Assuming that star A remained constant at the level listed by HM01, star B should have brightened accordingly from 18.88 to 17.29 in $B$, 18.10 to 16.69 in $V, 17.71$ to 16.31 in $R_{\mathrm{C}}$ and 16.92 to 15.27 in $I_{\mathrm{C}}$.

\section{Spectroscopy and kinematics}

The low-resolution, flux-calibrated spectrum of DT Ser presented in Fig. 3 was obtained on 25 Aug. 2011 under 2.5-arcsec seeing conditions, with the Asiago $1.22 \mathrm{~m}$ telescope $+\mathrm{B} \& \mathrm{C}$ spectrograph. The 2-arcsec-wide and 5-arcmin-long slit was centered on star A and oriented E-W, thus intersecting the distorted rim of the nebula. The slit missed star B, which was invisible on the telescope TV guiding system and, as expected, did not reveal itself during the data reduction. The sky background was traced from 12 to 22 arcsec away from star A in both direction, therefore well external to the planetary nebula and star B. The integrated fluxes of the emission lines are listed in Table 2.

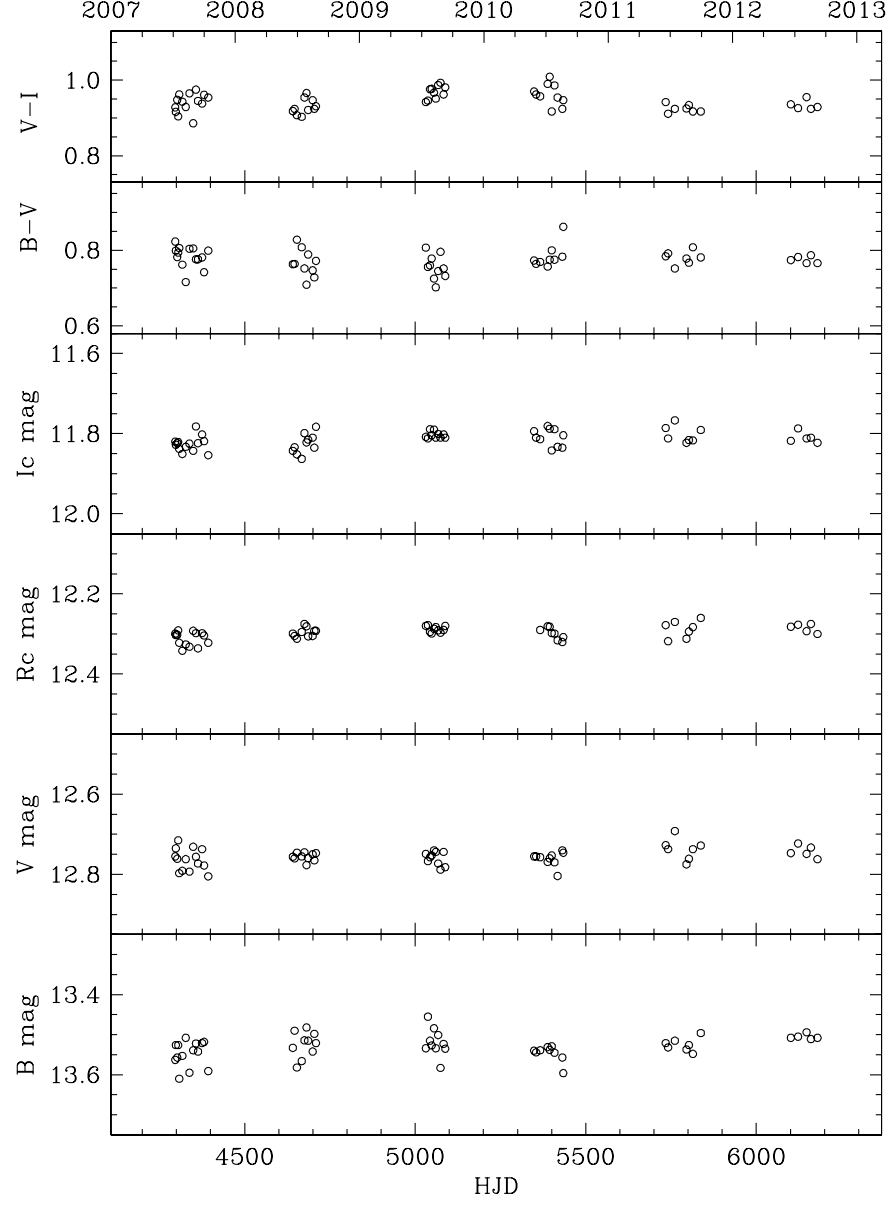

Fig. 2. Photometric evolution of DT Ser (data from Table 1). 

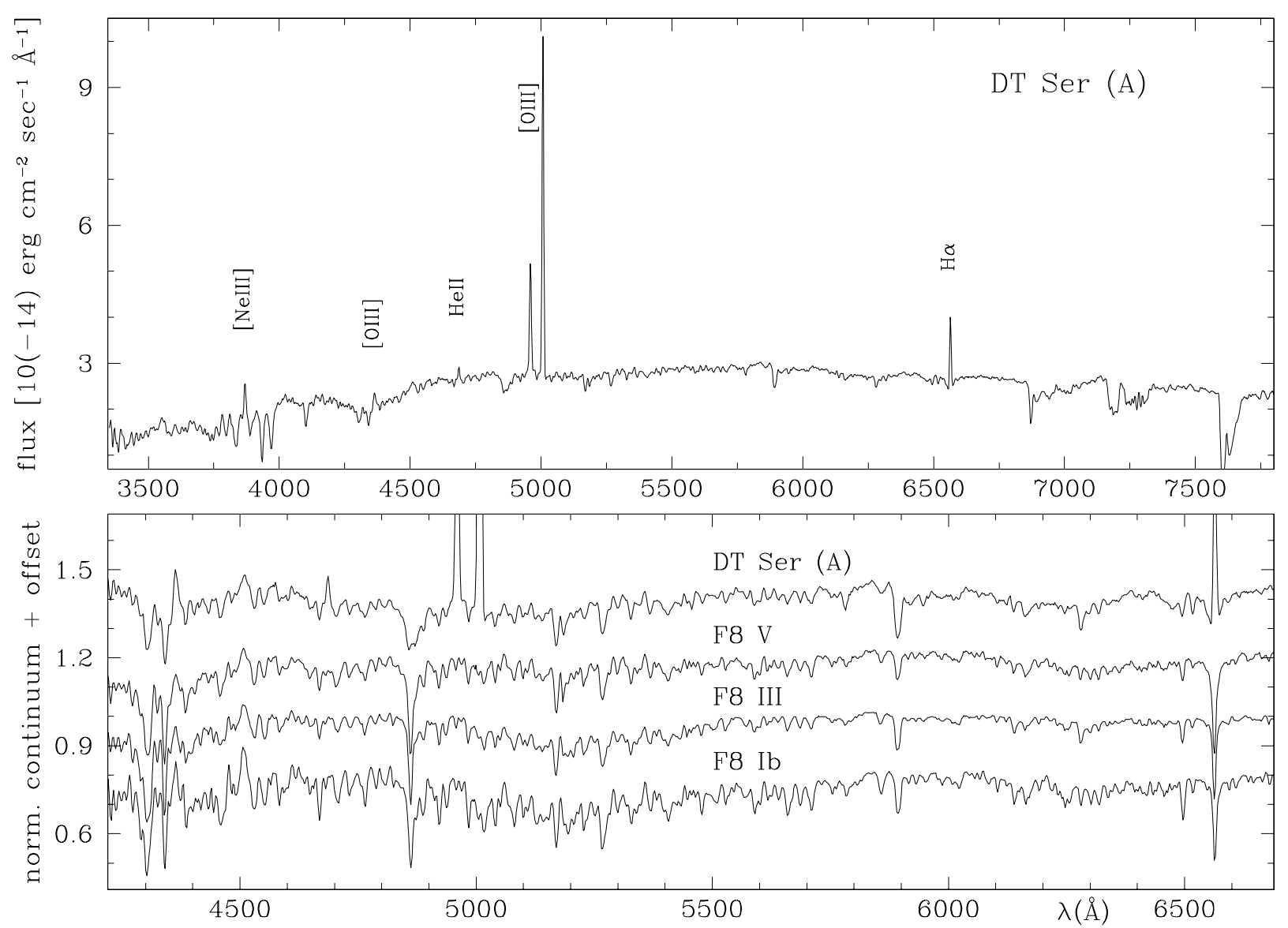

Fig. 3. Top: flux-calibrated spectrum of DT Ser (star A) for 25 Aug. 2011. Bottom: comparison of the spectral continuum of DT Ser from the upper panel with those of MKK standards HD 9826 (F8 V), HD 224342 (F8 III), and HD 45412 (F8 Ib).

Table 2. Integrated fluxes (observed and de-reddened by $E_{B-V}=0.20$ ) of the emission lines in the low-resolution spectrum of DT Ser presented in Fig. 3.

\begin{tabular}{cccc}
\hline \hline$\lambda(\AA)$ & \multirow{2}{*}{ Ion } & \multicolumn{2}{c}{ Flux $\left(\mathrm{erg} \mathrm{cm}^{-2} \mathrm{~s}^{-1}\right)$} \\
\cline { 3 - 4 } & & (obs.) & (de-redd. $)$ \\
\hline 6563 & $\mathrm{Ha}$ & $1.55 \times 10^{-13}$ & $2.47 \times 10-13$ \\
5007 & {$[\mathrm{OIII}]$} & $6.04 \times 10^{-13}$ & $1.18 \times 10-12$ \\
4959 & {$[\mathrm{OIII}]$} & $1.85 \times 10^{-13}$ & $3.60 \times 10-13$ \\
4686 & $\mathrm{HeII}$ & $2.25 \times 10^{-14}$ & $4.65 \times 10-14$ \\
4363 & {$[\mathrm{OIII}]$} & $4.49 \times 10^{-14}$ & $9.56 \times 10-14$ \\
3869 & {$[\mathrm{NeIII}]$} & $7.71 \times 10^{-14}$ & $1.80 \times 10-13$ \\
\hline
\end{tabular}

We have $\chi^{2}$ compared the continuum spectrum of star A with the complete MK spectral atlas obtained with the same Asiago $1.22 \mathrm{~m}$ telescope, spectrograph, and detector. When we masked the regions affected by the emission lines, the best match was found around spectral type $\sim$ F8. The fit is not satisfactory however, especially for the luminosity class that remains essentially undetermined, and seems to suggest that the absorption continuum of star A is not that of a normal star. The comparison of the absorption continuum of star A with those of MKK standards for types F8 V, F8 III, and F8 Ib is presented in Fig. 3.

Spatially resolved, high-resolution Echelle spectroscopy of star A and the surrounding nebula has been obtained on 27 Aug. 2012 with the Asiago $1.82 \mathrm{~m}$ telescope, under 2 arcsec seeing conditions. The spectrograph slit, widened to $2 \operatorname{arcsec}$ and providing a resolving power of 20000 , extended spatially for 30 arcsec. Star A was placed at the center of the slit and the telescope auto-guide tracked it. Four separate deep spectra were obtained with the slit rotated by $0^{\circ}, 45^{\circ}, 90^{\circ}$, and $135^{\circ}$ from the north-south alignment. Small portions of these spectra centered on [O III] $5007 \AA$ and H $\alpha$ are presented in Figure 1. They reveal the kinematical properties of the extended nebulosity, and in particular the hollow elliptical position-velocity structure typical of rims in planetary nebulae.

On each of the Echelle exposures, calibrated spectra were extracted tracing star A and two parallel positions separated by $\sim 4$ and $\sim 8$ arcsec on either sides of star A. A total of 20 spectra were obtained: four referring to star A, eight distributed across the planetary nebula, and another eight symmetrically placed on the other side of star A which were used to check the other spectra. The velocity profile of the $\mathrm{H} \alpha$, [O III] $5007 \AA$, and He II $4686 \AA$ emission lines at three representative locations of the planetary nebula are presented in Fig. 4. These three points (identified in Fig. 1) are aligned along the diameter of the nebula and avoid the bright star A. The heliocentric values of the photocentric radial velocity of the emission line profiles, averaged over the eight positions covering the extension of the planetary nebula, are $+37.7 \pm 0.5 \mathrm{~km} \mathrm{~s}^{-1}$ for $\mathrm{H} \alpha,+29.6 \pm 0.4 \mathrm{~km} \mathrm{~s}^{-1}$ for [O III] 5007 and $4959 \AA$, and $+34.0 \pm 1.0$ for He II (quoted uncertainties are the error of the mean). These differences reflect different formation regions for the different ions combined with the aspherical nebular geometry. The heliocentric radial velocity of the nebula, averaged over the various emission lines, is $+34 \mathrm{~km} \mathrm{~s}^{-1}$. 

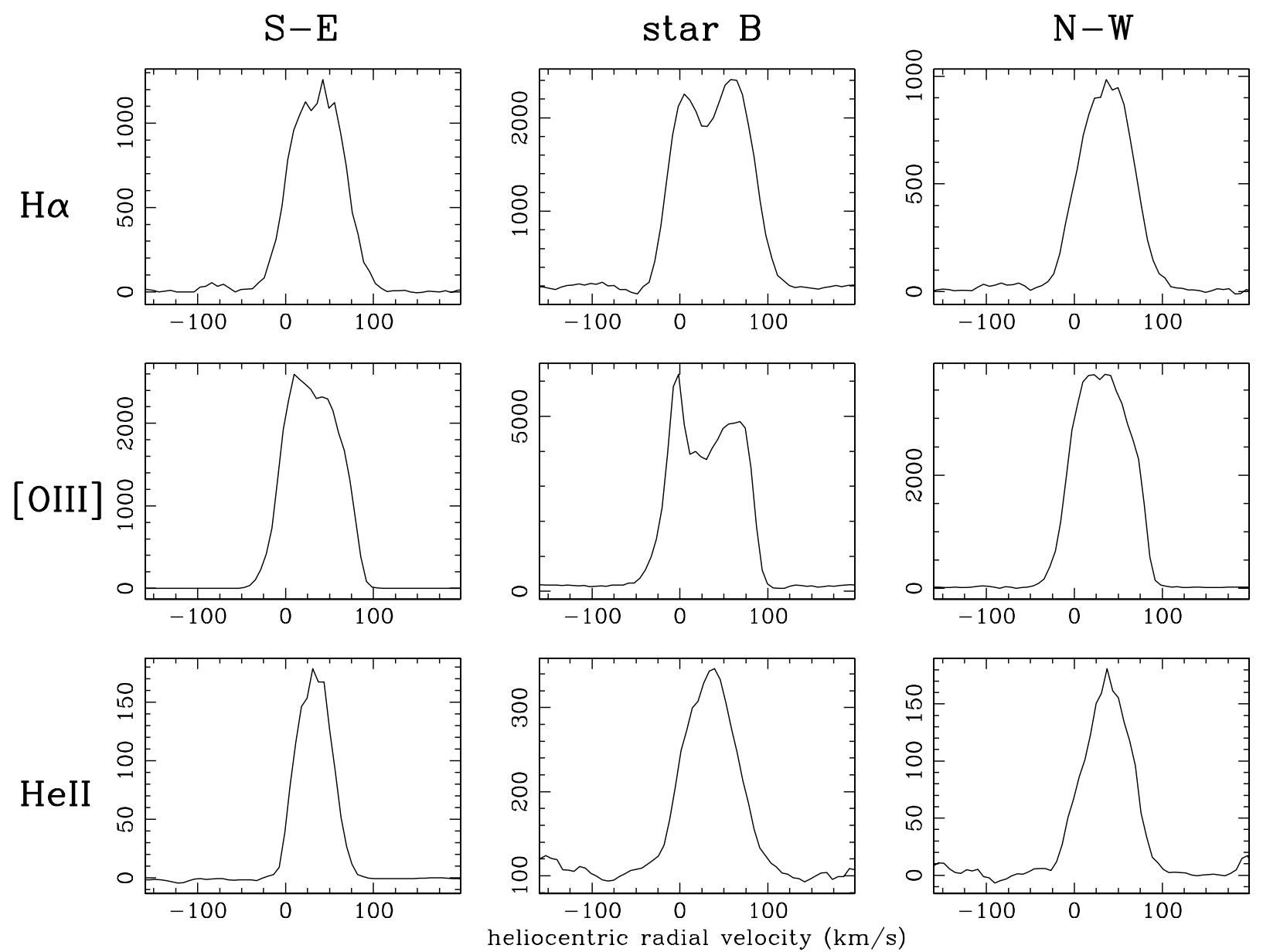

Fig. 4. Velocity profiles of the planetary nebula emission lines from spatially resolved Echelle spectroscopy (see sect. 5). The S-E, N-W, and star B positions are identified on the planetary nebula image at the top-left corner of Fig. 1.

The systemic velocity of the nebula calculated from the central wavelength (mean value of the two peaks) of the $\mathrm{H} \alpha$ split emission line where the spectrum crosses the central star (star B) is similar, $+33 \mathrm{~km} \mathrm{~s}^{-1}$. The heliocentric radial velocity of star A, derived from its absorption spectrum is $-26.7 \pm 0.8 \mathrm{~km} \mathrm{~s}^{-1}$.

The emission line profiles observed at the center of the nebula (labeled star B in Fig. 4) suggest that the expansion velocity within the nebula increases with distance from the central star. The He II line, forming closer to the central star, is the sharpest and displays a Gaussian profile like that expected from a filled spherical gas distribution. The $\mathrm{H} \alpha$ line, forming in a volume of space larger than the He II region, is broader than the He II line and displays a double-peaked profile with a velocity separation of $55 \mathrm{~km} \mathrm{~s}^{-1}$. [O III], forming preferentially in the lower density outer regions, shows an even larger velocity separation between the peaks, about $75 \mathrm{~km} \mathrm{~s}^{-1}$.

\section{Discussion}

\subsection{Reddening}

The reddening that affects star A can be derived from HM01 $U B V R_{\mathrm{C}} I_{\mathrm{C}}$ and $2 \mathrm{MASS} J H K_{\mathrm{s}}$ photometry, and the intrinsic colors for F8 stars taken from Fitzgerald (1970), Bessell (1990), and Straizys (1992). The result is $E_{B-V}=0.19 \pm 0.02$. A similar amount can be derived by simple geometry. The reddening at the Galactic poles is $E_{B-V} \sim 0.036$ and the dust layer extends for $\sim 140 \mathrm{pc}$ on both sides of the the Galactic plane (averaging the many determinations found in literature). The corresponding total reddening integrated toward star $\mathrm{A}$ is $E_{B-V}=0.20$ for a Galactic latitude $b=+10.3$ and a line of sight that traverses the whole dust layer.

\section{2. $\operatorname{Star} A$}

Assuming a reddening $E_{B-V}=0.19$ and a spectral type F8, the distance to star A is 400 pc for a star of luminosity class V, $1200 \mathrm{pc}$ for class III, and $22 \mathrm{kpc}$ for class Ib, adopting the HIPPARCOS scale of absolute magnitudes calibrated by Sowell et al. (2007). The $61 \mathrm{~km} \mathrm{~s}^{-1}$ difference between the radial velocity of star A and the planetary nebula surrounding star B indicates that the two are not related, unless the single-epoch radial velocity we measured for star A is perturbed by a largeamplitude orbital motion around an unseen companion.

There are several puzzling features however that should not occur if star A were a normal field star unrelated to star B and the nebula.

First, as noted in Sect. 3, the rim of the planetary nebula is severely distorted. Its $\mathrm{SE}$ side is brighter and closer to the central star, while the NE part is fainter and elongated toward star A, giving the impression that it might be related to it.

Second, with normal field stars we do not usually experience such a poor $\chi^{2}$ spectral fitting to primary MKK standard stars as we obtained for star A, suggesting a peculiar nature and/or chemical partition. 
Third, the WISE satellite medium-infrared all sky survey lists a bright source (WISE J180152.26-012615.8) coincident with star A with magnitudes $\mathrm{W} 1=10.637(3.35 \mu \mathrm{m})$, $\mathrm{W} 2=10.616(4.6 \mu \mathrm{m}), \mathrm{W} 3=8.872(11.6 \mu \mathrm{m})$, and $\mathrm{W} 4=4.071$ $(22.1 \mu \mathrm{m})$. This large excess in the $\mathrm{W} 3$ and $\mathrm{W} 4$ bands indicates the presence of a detached, cold-dust circumstellar shell around star A. The high spatial accuracy of the WISE satellite astrometry (0.06 arcsec on both coordinates, which we confirmed by verifying the coincidence of WISE, 2MASS, and PPMXL coordinates for many field stars around DT Ser), rules out the possibility that the WISE source could either be star B or the planetary nebula. Such a cold, detached dust shell is typically associated with post-AGB objects.

If star $\mathrm{A}$ is indeed a post-AGB star, this could account for several peculiarities we found. (a) The $-61 \mathrm{~km} \mathrm{~s}^{-1}$ difference in radial velocity with the planetary nebula. In post-AGB stars, the pseudo-photosphere forms in the outflowing wind, which causes a blueshift of the observed radial velocity. An outflow velocity of about one hundred $\mathrm{km} \mathrm{s}^{-1}$ is common in post-AGB stars. (b) The difficulty in classifying the spectrum of star A. Absorption lines forming in a fast outward-moving medium do not obey to the same curve-of-growth as those forming in the nearly static atmospheres of normal stars. The chemical partition is also expected to be markedly different, because of the exposure of nuclearly processed internal layers after the hydrogen-rich outer envelope has been blown away. (c) The discrepancy between the results of Bond (1978), who saw Balmer lines of star A in emission, and those of Cieslinski et al. (1997) who instead found them in absorption. Emission lines are known to vary in post-AGB stars, depending primarily on the intensity of the variable wind.

\subsection{The planetary nebula}

The emission lines from the high excitation ([O III] $\gg \mathrm{H} \alpha$ ) planetary nebula are very bright, and their spatial extension away from the stellar image is quite obvious, as Fig. 1 shows. How could it be that neither B78 nor C97 noticed them? Especially puzzling is the non-detection by $\mathrm{C} 97$ who observed under good seeing conditions and exposed separately on stars A and B. An unorthodox speculation would be to assume that the surface brightness of the planetary nebula may have varied with time. This could happen in response to varying photo-ionization input from the central star. The timescale of the response from the nebula is the recombination time, which is related to the electron density.

The critical density for nebular [O III] lines, which are highly pronounced in DT Ser, is $\log N_{\mathrm{e}}=5.8 \mathrm{~cm}^{-3}$, that for the missing [N II] lines is $\log N_{\mathrm{e}}=4.9 \mathrm{~cm}^{-3}$. The [O III] ratio $(5007+4959) / 4363=17.6$ from Table 2 confirms that the nebula has a very high density, namely $\log N_{\mathrm{e}} \geq 5.64 \mathrm{~cm}^{-3}$ assuming an electron temperature $T_{\mathrm{e}} \leq 20000 \mathrm{~K}$ as typical of photoionized nebulae (Osterbrock \& Ferland 2006). Such electron density is one or two orders of magnitude higher than commonly found in planetary nebulae (Stanghellini \& Kaler 1989). A much higher electron temperature, such as encountered in shock fronts, would lower the electron density $\left(\log N_{\mathrm{e}}=4.0 \mathrm{~cm}^{-3}\right.$ for $T_{\mathrm{e}}=$ $40000 \mathrm{~K})$. The electron density estimate is relevant for the total mass derived for the nebula. Approximating the planetary nebula with a sphere uniformly filled with hydrogen, its mass would scale with distance $D$ and filling factor $\xi$ as

$$
M_{\mathrm{PN}}=0.55 \xi\left(\frac{D}{\mathrm{kpc}}\right)^{3} M_{\odot} .
$$

The typical mass of planetary nebulae (Boffi \& Stanghellini 1994) is few a tenths of a solar mass, while that of symbiotic stars is one or two orders of magnitude smaller, with the notable exception of Hen 2-104 which is as high as $\sim 0.1 M_{\odot}$ (Santander-García et al. 2008). This would suggest a distance to DT Ser not much larger than $1 \mathrm{kpc}$ for $\xi=0.4$, a value typical of planetary nebulae (Boffi \& Stanghellini 1994).

Assuming $\log N_{\mathrm{e}}=5.4 \mathrm{~cm}^{-3}$, the recombination timescale for hydrogen of planetary nebula in DT Ser is (Ferland 2003)

$t_{\mathrm{rec}}=0.66\left(\frac{T_{\mathrm{e}}}{10^{4} \mathrm{~K}}\right)^{0.8}\left(\frac{n_{\mathrm{e}}}{10^{9} \mathrm{~cm}^{-3}}\right)^{-1} \approx 85$ days,

which is shorter than the timescale between the sparse observations available of DT Ser, and therefore compatible with possile significant variation of the surface brightness of the planetary nebula in between them.

Some studies have found by combining radio and optical data (e.g. Lee \& Kwok 2005; Vázquez et al. 1999) that a nonuniform internal distribution of dust can play an important role in the morphology of some PN. The amount of dust associated with the PN in DT Ser seems negligible because of two reasons. The sensitive WISE mid-infrared survey did not detected any emission associated to the $\mathrm{PN}$, and no $\mathrm{Na}$ I doublet absorption line is observed at the systemic velocity of the PN on our Echelle spectrum of star A. This spectrum has a signal-to-noise of 92 on the continuum around the Na I doublet at 5890 and $5896 \AA$, and the upper limit of the equivalent width of any PN-related component is $0.006 \AA$. Adopting the calibration of Munari \& Zwitter (1997), which was derived for the diffuse interstellar medium, this translates into un upper limit of $E_{B-V}<0.002$. If star $\mathrm{A}$ is related to the planetary nebula and it is seen through the outer layer of the nebula, the $E_{B-V}<0.002$ limit suggests a negligible amount of dust associated to the PN and located along the line of sight to star A.

\subsection{Star B}

The planetary nebula we have discovered shows widespread He II emission, indicating a hot central ionizing source: to support production of He II via photo-ionization, the WD must have a temperature $T_{\text {eff }}>55000 \mathrm{~K}$ (Allen 1984). The optical photometric colors of very hot stars do not strongly depend on their actual temperature, their effective wavelengths being on the Rayleigh-Jeans tail of the energy distribution: for an unreddened very hot star we may assume, according to Straizys (1992) and Drilling \& Landolt (2000), $U-B=-1.20, B-V=-0.35$ and $V-I_{\mathrm{C}}=-0.34$. These colors cannot be simultaneously reconciled with those observed for star B $(U-B=-0.71$, $B-V=+0.44$ and $V-I_{\mathrm{C}}=+0.05$, average of C97 and HM08 data) for any assumed reddening. A compromise could be to settle for $E_{B-V} \sim 0.52$, which is appreciably higher than the interstellar reddening derived in Sect. 6.1 above. This suggests that star B is a binary system in which the ionizing source has a cooler companion that softens the combined optical energy distribution toward cooler temperatures, as in symbiotic binaries. If the companion is a star with a strong wind, part of it should be ionized by the hot star, producing emission lines. No sharp emission line component is observed to stand over the planetary nebula diffuse emission at the position of star B in the highresolution Echelle spectra of Fig. 1.

The most puzzling aspect of star B is its variability, which is not expected from the central star of a normal planetary nebula. The C97 and HM08 photometry of star B, separated in time by 
four years, differs by $0.8 \mathrm{mag}$ in $V$. It has to be remarked that C97 performed aperture photometry, while HM08 carried out PSF fitting because of the close proximity of stars A and B. Some light leak from star A could have contaminated the measurement of star B by Cieslinski et al. (1997), but with the available information we cannot estimate if this indeed occurred, and if so, to what extent. B78 reported that during acquisition of his spectra, star B appeared only slightly fainter than star A. When we were exposing our spectra, star B was so much fainter than A as to be undetectable on the TV guiding systems of both the Asiago $1.22 \mathrm{~m}$ and $1.82 \mathrm{~m}$ telescopes.

Without doubts, the brightness of the combined star A+B image does indeed change with time, as outlined in Sects. 2 and 4 above. The steady increase in brightness in Fig. 2 continued for five years. If this is related to the changing orbital aspect, the orbital period should be $\geq 10$ years. This would resemble the lowamplitude ( $\Delta V=0.14 \mathrm{mag}), 16.8 \mathrm{yr}$ orbital modulation of the yellow symbiotic star V471 Per, which harbors a G5 III donor star (Munari et al. 2012b).

\section{Concluding remarks}

The whole picture surrounding DT Ser is both puzzling and intriguing, and the object is far more interesting and exciting than a normal symbiotic star. If there really is a symbiotic binary in the system, its association with a planetary nebula is the first such known case. If not, the large photometric variability observed for the nucleus of the planetary nebula is nevertheless a rarity, if not a unique case. On top of all this, the bright star A may also be a post-AGB star. At $5 \mathrm{kpc}$ distance, the 5 arcsec angular separation translates into a spatial separation of $0.1 \mathrm{pc}$, the typical radius of a planetary nebula.

A firm conclusion about the true nature of DT Ser, the planetary nebula, and the two stars A and B needs additional devoted observations. In particular, it seems relevant to obtain (a) multiepoch photometry and spectroscopy of the spatially resolved star B to confirm its variability and look for a signature of a companion; (b) deep imaging at higher spatial resolution of the planetary nebula, especially the part closest to star A; (c) an atmospheric analysis of star A, to determine the distance (from a comparison of the derived $T_{\text {eff }}, \log g$, and $[\mathrm{M} / \mathrm{H}]$ with stellar isochrones) and the evolutionary status (from chemical abundances); (d) epoch radial velocities of star A to examine whether if the radial velocity difference with the planetary nebula may be due to orbital motion (around an unseen companion in a close orbit) or absorption lines forming in a optically thick wind, such as that of a post-AGB star.
Acknowledgements. Based in part on observations made with the Nordic Optical Telescope, operated jointly by Denmark, Finland, Iceland, Norway, and Sweden, on the island of La Palma in the Spanish Observatorio del Roque de los Muchachos of the Instituto de Astrofísica de Canarias. R.L.M.C. acknowledges funding from the Spanish AYA2012-35330 grant.

\section{References}

Allen, D. A. 1984, PASA, 5, 369

Belczyński, K., Mikołajewska, J., Munari, U., Ivison, R. J., \& Friedjung, M. 2000, A\&AS, 146, 407

Boffi, F. R., \& Stanghellini L. 1994, A\&A, 284, 248

Corradi, R. L. M. 2003, in Symbiotic stars probing stellar evolution, eds. R. L. M. Corradi, J. Mikolajewska, \& T. J. Mahoney, ASP Conf. Ser., 303, 393

Corradi, R. L. M., Ferrer, O. E., Schwarz, H. E., Brandi, E., \& García, L. 1999, A\&A, 348, 978

Corradi, R. L. M., Livio, M., Balick, B., Munari, U., \& Schwarz, H. E. 2001a, ApJ, 553, 211

Corradi, R. L. M., Munari, U., Livio, M., et al. 2001b, ApJ, 560, 912

Corradi, R. L. M., Balick, B., \& Santander-García, M. 2011, A\&A, 529, A43

Drilling, J. S., \& Landolt, A. U. 2000, Allen's Astrophysical Quantities, 4th edn., ed. A.N. Cox (AIP Press Springer), 381

Ferland, G. J. 2003, ARA\&A, 41, 517

Guerrero, M. A., Chu, Y.-H., \& Miranda, L. F. 2004, AJ, 128, 1694

Henden, A., \& Munari, U. 2000, A\&AS, 143, 343

Henden, A., \& Munari, U. 2001, A\&A, 372, 145

Henden, A., \& Munari, U. 2006, A\&A, 458, 339

Jurdana-Šepić, R., \& Munari, U. 2010, PASP, 122, 35

Lampland, C. O. 1922, PASP, 34, 218

Lee, T.-H., \& Kwok, S. 2005, ApJ, 632, 340

Munari, U., \& Moretti, S. 2012, Balt. Astron., 21, 22

Munari, U., \& Patat, F. 1993, A\&A, 277, 195

Munari, U., \& Zwitter, T. 1997, A\&A, 318, 269

Munari, U., Bacci, S., Baldinelli, L., et al. 2012a, Balt. Astron., 21, 13

Munari, U., Siviero, A., Tamajo, E., et al. 2012b, Balt. Astron., 21, 180

Ostrebrock, D. E., \& Ferland, G. J. 2006, Astrophysics of Gaseous Nebulae and Active Galactic Nuclei (Univ. Science Books)

Paresce, F., \& Hack, W. 1994, A\&A, 287, 154

Santander-García, M., Corradi, R. L. M., Balick, B., \& Mampaso, A. 2004, A\&A, 426, 185

Santander-García, M., Corradi, R. L. M., Whitelock, P. A., et al. 2007, A\&A, 465,481

Santander-García, M., Corradi, R. L. M., Mampaso, A., et al. 2008, A\&A, 485, 117

Schwarz, H. E. 1991, A\&A, 243, 469

Schwarz, H. E., \& Corradi, R. L. M. 1992, A\&A, 265, L37

Schwarz, H. E., Aspin, C., \& Lutz, J. H. 1989, ApJ, 344, L29

Schmeja, S., \& Kimeswenger, S. 2001, A\&A, 377, L18

Schönberner, D., Jacob, R., Steffen, M., et al. 2005, A\&A, 431, 963

Solf, J. 1983, ApJ, 266, L113

Solf, J. 1984, A\&A, 139, 296

Solf, J., \& Ulrich, H. 1985, A\&A, 148, 274

Sowell, J. R., Trippe, M., Caballero-Nieves, S. M., \& Houk, N. 2007, AJ, 134, 1089

Stanghellini L., \& Kaler, J. B. 1989, ApJ, 343, 811

Vázquez, R., Torrelles, J. M., Rodríguez, L. F., et al. 1999, ApJ, 515, 633 